\title{
Recent advances on surface modification of halloysite nanotubes for multifunctional applications
}

\author{
Yongtao Yang, Yun Chen, Fan Leng, Li Huang, Zijian Wang, Weiqun Tian* \\ Department of Biomedical Engineering, School of Basic Medical Sciences, \\ Wuhan University, Wuhan 430071, China. \\ *Corresponding author’s E-mail: tian_weiqun@whu.edu.cn
}

\begin{abstract}
Halloysite nanotubes (HNTs) are natural occurring mineral clay nanotubes that have excellent application potential in different fields. However, HNTs are affected by size effect, surface electron effect and hydrogen bond formation on the surface which lead to weak affinity and prone to reunion at some extent. It is very significant to modify the HNTs' surface for expand its applications. In this review, the structural characteristics, performance and the related applications of surface modification HNTs are reviewed and summarized. we focus on the surface modified methods of HNTs, the effect of surface modification on materials and its related applications in various regions. In addition, future prospects and the meaning of surface modification have been discussed in HNTs studies. This review provided a reference for the application of HNTs modifications in new fields.
\end{abstract}

Keywords: halloysite nanotubes; surface modification; structural characteristics; application

\section{Introduction}

HNTs are natural occurring mineral clay nanotubes with particular hollow shapes. There are various morphologies for HNTs, such as tubes, platy and spheres [1], and with $500 \mathrm{~nm}-1500 \mathrm{~nm}$ length, the lumen and external diameter respectively $15 \mathrm{~nm}$ and $50 \mathrm{~nm}$ [2]. HNTs possess a high surface area reached to $184.9 \mathrm{~m}^{2} / \mathrm{g}$ and large pore volume at $0.353 \mathrm{~cm}^{3} / \mathrm{g}$ [3] to form matrix membrane for gas separation [4]. HNTs chemical composition is similar to kaolin. However, the unit layers are isolated by monolayer water molecules in HNTs. The HNTs hold the molecular formula of $\mathrm{Al}_{2} \mathrm{Si}_{2} \mathrm{O}_{5}(\mathrm{OH})_{4} \cdot \mathrm{nH}_{2} \mathrm{O}$ [5] and the HNTs is composed of $\mathrm{Al}, \mathrm{O}$ and $\mathrm{Si}$ with the atomic 
proportion 1:4.6:1 [6]. The aluminosilicate clay nanotubes have Al:Si ration with 1:1. There are two main polymorphs for HNTs anhydrous form and hydrated form, interlayer spacing respectively $7 \AA$ and $10 \AA$ [7]. HNTs have a wide range of $\mathrm{pH}$ and the zeta-potential shown a negative electrical with ca $-50 \mathrm{mV}$ [8]. At $\mathrm{pH}$ 6-7, the zetapotential of HNTs put up negative but exerts a positive surface at $\mathrm{pH} 8.5$ [9] and a negative charge with ca $-32 \pm 2 \mathrm{mV}$ [10]. The external surface of halloysite nanotubes is composted silicon oxygen tetrahedron, the internal lumen is alumina oxygen octahedron, outer surface is the mainly distribution of Si-O-Si group, the inner surface is Al-OH [11]. Because of the multilayer structure, most of the hydroxyl exists within the lumen and only a few in the outer surface [12].

As a widely used environmentally friendly clay tubes, HNTs have good biocompatibility [13]. HNTs were confirmed have non-toxic not only in vivo [10], but also in vitro [14]. HNTs with High specific surface area, strong surface adsorption and different chemistry of the inner and outer surface. However, HNTs showed a weak affinity when used to synthesize composites, drug delivery and molecular adsorbents, because of the weak intermolecular force, like Van der Waals forces and hydrogen bonding. To improve the performance of HNTs, a surface modification is very ideal. For example, the modified HNTs can be used as nanofillers to composite polymer to enhance mechanical strength [15] and as nanocarries to implement sustain drug delivery. In addition, it is also be regarded as adsorbent to absorb or remove the matter from aqueous solution [16] and served as catalysts [17] to the study of reaction.

\section{Surface modification of HNTs and the relevant properties}

Surface modification of HNTs means that it maintains the original properties and endows the new properties, such as hydrophilicity, biocompatibility, antistatic properties, dyeing performance. At present, many methods of surface modification of HNTs are reported, which can be attributed to surfactant modification, coupling agent modification, intercalation modification, surface coating modification and free radical modification. The HNTs can be modified in the appropriate time according to the need for selective modification.

\subsection{Surfactant modification}

Surfactant modification refers to the presence of non-polar lipophilic groups and polar hydrophilic groups in the surfactant molecule. When the surfactant binds to the nanotubes, it has a better dispersion. Yong Lin et al (2011) [18] prepared the highimpact polystyrene nanospheres by emulsion polymerization. In this system, the sodium dodecyl sulfate (SDS) was added to the aqueous solution containing the HNTs. SDS as an emulsifier to form a molecular layer on the surface of HNTs, so that the surface of HNTs has a strong hydrophilic and enhance the dispersion in aqueous solution. In addition, Wang et al (2010) [19] used the surfactant of hexadecyltrimethylammonium bromide (HDTMA) to modify the HNTs and prepared a new 
adsorbent for the removal of $\mathrm{Cr}$ (VI) from the aqueous solution, and the adsorbent with the maximum adsorption rate of $\mathrm{Cr}(\mathrm{VI})$ reached to $90 \%$ in the 5 minutes.

\subsection{Coupling agent modification}

Grafted silane coupling agent onto the surface was the most common chemical modification method for HNTs. The silane coupling agent can be chemically reacted with the HNTs by physical or chemical bonding. By changing the performance of hydrophobicity, to improved the dispersibility and interfacial interaction between HNTs and polymers. Guo et al (2009) [20] synthesized a high strength nanocomposite (polyamide 6/halloysite) by combined with 3-(trimethoxy silyl) propyl methacrylate. The results showed that the nanocomposites significantly improve mechanical and thermal properties. Meanwhile, Wan et al (2017) [21] prepared a kind of highperformance nanocomposites by compounded with 3-aminophenoxy-phthalonitrile and poly (arylene ether nitrile) (PEN) based on HNTs. It has been found that functionalized HNTs exhibit superior tensile strength and modulus, Because of the better dispersion and strong capacitance.

\subsection{Intercalation modification}

Intercalation modification refers to the small molecules reacted withHNTs between the hydroxyl groups and other groups, in order to improve the performance of HNTs. Tang et al (2011) [22] used the phenylphosphonic acid (PPA) to unfold and intercalate the HNTs, and mixed with epoxy to form the halloysite-epoxy nancomposites. The modified HNTs obtained a better dispersion, large contact area among nanocomposites and significantly promoted the micro-cracks and plastic deformation take shape at the interface. Deng et al (2009) [23] treated the HNTs with potassium acetate (PA) and ball mill homogenisation to improved particle dispersion. It was demonstrated that the treatment HNTs could observably enhanced the properties of mechanical, interfacial debonding and provided opportunities for other substances to intercalate.

\subsection{Surface coating modification}

Surface coating modification refers to the surface of HNTs is coated with a layer of polymer or inorganic material by means of the electrostatic adsorption, so as to achieve the purpose of changing HNTs performance. Li et al. (2016) [24] prepared the drug-loaded porous microspheres (Hal-CTS/Asp) by the method of thoroughly emulsification in the water/oil microemulsion. The HNTs were coated with chitosan (CTS) and the aspirin (Asp) adsorbed inside the microspheres as a model drug. The results indicated that the microspheres had the characteristics of high surface area and the large-interconnected pores, which was conducive to the adsorption of aspirin. The modified HNTs had excellent loading capacity (42.4 wt \%) nearly twenty times higher than unmodified ones $(2.1 \mathrm{wt} \%)$. Meanwhile, the special microspheres showed low 
drug release rate and $\mathrm{pH}$ sensitive compared with the pristine HNTs. Liu et al (2015) [25] successfully prepared a lot of alginate/HNTs composites tissue engineering scaffolds by electrostatic adsorption method. The scaffolds showed significant enhancement in thermal stability and cell-attachment properties.

\subsection{Free radical modification}

The surface of HNTs contains hydroxyl groups that could react with the monomer on the inner or outer surface. The functionlization HNTs can improve the hydrophobicity and their dispersibility in organic solvents, in order toobtain the stable composites. Liu et al (2011) [26] prepared the modified HNTs by grafted the polymethyl methacrylate(PMMA) via radical polymerization, and then compounded with poly(vinyl chloride) (PVC) to form the high toughness, strength and modulus composites. The results showed that the modified HNTs have an uniform dispersed in PVC aqueous solution, and effectively improve the mechanical properties. Li et al (2008) [27] reported a kind of functionlization HNTs modified by polymers via atom transfer radial polymerization (ATRP) and crosslinked with polystyrene (PS) and polyacrylonitrile (PAN), respectively. The results indicated that the composites showed excellent wettability and can be used to entrap water droplets.

\section{Application of Surface Modification of HNTs.}

\subsection{As the filler nocomposites.}

Composite materials are vital for the development of modern science and technology. They are widely used in magnetic material, magnetic facility, flame retardant, optics, scaffold for tissue engineering and electronics. But these nanocomposites always need a complex template, tedious preparation process and high cost. To find an effective modules and efficient fabrication will be imperative.

Due to high specific surface area and unique surface chemical properties, HNTs widely used to improve polymer's property. At the meanwhile, the low surface charges and weak interfacial could be problematic [28].

But if it is modification on the surface of HNTs to spread the basal spacing through insert the inorganic or organic groups between layers. The surface modified HNTs not only acquire well dispersibility and strong interfacial interaction [29], but also provide abundant bond formation [30]. The functionalization of nanotubes composite polymer will achieve a win-win situation.

HNTs have been used more and more for enhacing properties of ploymers. Parthajit et al (2013) [5] successful modified the HNTs by N-(b-aminoethyl)-caminopropyltri-methoxysilane, the modification and unmodification respectively mingle with nonpolar polypropylene (PP) and polar polyoxymethylene (POM) by utilizing the methods of immiscible blend system(B). The results indicate that pure polymer blend and B-HNT nanocomposites always form obvious agglomeration attribute to the weak interface interaction between the polymer and HNTs. Howeve it 
present different phenomenon to the B-MHNT nanocomposites that dispersed well in the polymer blend. This suggests that modification (B-MHNTs) obtain a better dispersion compared to the unmodified (B-HNTs) in blend matrix. Meanwhile, the functionalization HNTs are used to enhance the chemical interactions as a natural rubber (NR) filler [31]. The bis (triethoxysilylpropyl)-tetrasulphide was used to modified the HNTs by the way of silane coupling agent. It can be summarized that the natural rubber composite with modified HNTs (NR-HNTs-Si) show an excellent physical properties and thermal stability compared with the unmodified HNTs nanocomposite (NR-HNTs) and natural rubber-silica (NR-Si). The HNs also modified with polyrhodanine (PRD) by the way of oxidative polymerization to Styrene butadiene rubber (SBR) [32]. The data indicate that the tensile strength and SBR/PRD-HNTs composites which PRD-HNTs composite SBR have significant reinforce compared with unmodified HNTs increased by $117 \%$ and $87 \%$, respectively. HNTs also can be treated with the $\gamma$-irradiation [33] to enhance the strength of epoxy nanoconposites. Comparing with untreat ones, the treatment have significant effective such as tensile strength and Young's modulus increasing $46 \%$ and $38 \%$ respectively. Because of the uniform dispersion, abundant hydroxy and chemical interaction .

\section{2 As the nanocarriers for drug delivery}

HNTs are environmentally friendly natural nanomaterials and low cost. With high porosity,adjustable surface chemistry structure [34], good biocompatibility [35] the large surface area, HNTs have great development prospects in the field of drug capacity with a sustained manner. Thus made it attracted a great deal of interest in biological medicine, biological science and technology. HNTs can be used as a multipurpose excipient that improved stability for sustained release of drugs [36].

It possess a special periodic multilayer with gibbsite octahedral $(\mathrm{Al}-\mathrm{OH})$ in internal surface and siloxane (Si-O-Si) on external surface [37]. HNTs have great application value in alternative modification with organic and inorganic functional molecules at diverse surface. The modified HNTs always obtain a better effect of drug loading than unmodified ones. Weng et al. (2012) [38] used octadecylphosphonic acid (ODP) modified halloysite nanotubes (halloysite-ODP) to load ferrocene with crosslinking method. The results showed that halloysite-ODP exerts more ascolloidal stability in the aqueous suspension than the unmodified HNTs. Comparing with HNTs, the halloysite-ODP possessed higher adsorption capacity and faster assimilate for hydrophobic molecules of ferrocene. There is a initial burst release for unmodified HNTs because of the inadequateinadequate between HNTs and ferrocene. But halloysite-ODP show a two-step release with a non-Fickian model. 
Besides, the HNTs modified with $\gamma$-aminopropyltriethoxysilane ( $\gamma$-APTES) could enhance the ability of loading analgesic [39]. The results demonstrated that the modified HNTs were more than three times higher capacity compared to unmodified HNTs. Furthermore, the modified HNTs have a long time sustaining release reached to $115 \mathrm{~h}$. In addition, the functionlization HNTs crosslink with the APTES used to load ibuprofen [40], for low loading capacity and burst release for HNTs. The date showed that the modified HNTs possess higher capacity to load ibuprofen increasing by $25.4 \%$ [41]. The release of ibuprofen indicated that the modified and unmodified HNTs put up a two-step release in vitro. However the modified HNTs showed slower release than unmodified ones due to strong electrostatic interactions.

\subsection{As the adsorbent}

As research point out that HNTs are natural occurring hollow tubes, within 10$150 \mathrm{~nm}$ diameter, 500-1500nm length and with large specific surface area and high aspect ratio [42]. The primary hydroxyl groups exist inexternal surface provided convenience for the HNTs experiment with some organics. These special properties made nanotubes have extensive applications for separation and absorption material in industrial extraction to enhance the ability of absorb various metal ions (Peng et al., 2010) [43]. Ruijun et al (2012) [44] used two-step modification methods to synthesize functionalized HNTs respectively react with APTES and murexide $(\mathrm{Mu})$. The results indicate that HNTs-Mu were ten times absorption higher than original HNTs for $\mathrm{Pb}$ (II) at $\mathrm{pH}$ 1. The phenomenon shown that the HNTs-Mu uptake Pd (II) and provide available sites for anionic metal complexes. The functionalization HNTs also used to adsorb Cr (VI) and remove it from aqueous solution (Wang et al., 2010) [45]. In the work, the functionalization HNTs were successful prepared by crossed with HDTMA. The results showed that the modified HNTs adsorbed nearly ninety percent of $\mathrm{Cr}$ (VI) within five minutes from aqueous solution with a Langmuir model. Meanwhile, the halloysite nanotubes modified with 2-methacryloyloxyethyl phosphorylcholine (MPC) utilized to adsorb BSA with the method of phase inversion [46]. The modified HNTs of absorb capacity increased $87 \%$ compared with the pure membrane.

As we all known, Zearalenone has a strong toxicity damage to the reproductive system. It is necessary to remove the toxicant for the development of animals. The fodder also adopts the modified HNTs to adsorb Zearalenone in the sow reproduction and piglet growth stage [47]. The HNTs were modified with stearyldimethylbenzylammonium chloride (SKC), the results demonstrated that the functionalization HNTs conspicuous reduced the damage compared with Zearalenonetreated one in the aspect of colostrum and milk $(\mathrm{p}<0.05)$. The vivo test results show 
that the modified HNTs possessed superior adsorb property than the unmodified ones for Zearalenone [48], and it can be summarized that in the gastrointestinal tract the modified HNTs have obvious composite ability with Zearalenone than the HNTs.

\subsection{As the catalysts}

There is no doubt that the rapid and efficient production is particularly important for the production. With the development of the industry, catalyst has been widely used to change the reaction rate [49] in the industry. The modified HNTs were used as catalyst, due to their large special surface area, high-activity and luxuriant surface hydroxyl groups [50]. The HNTs could be modified and synthesized the catalyst composites [51].

It is reported that the HNTs were modified with APTES and $\mathrm{HCl}$ to prepare a mod functionlization HNTs $(\mathrm{HNTs}-\mathrm{NH} 2 \cdot \mathrm{HCl})$ as metal nanoparticles to product $\mathrm{H}_{2}$ [52]. And the results point out that the $\mathrm{HRG}$ values of $\mathrm{HNTs}-\mathrm{NH}_{2} \cdot \mathrm{HCl}$ catalyst obtain a higher reaction than the HNTs catalyst with the value $813.08 \mathrm{~mL} \mathrm{~min}^{-1} \mathrm{~g}^{-1}$ catalyst and $630.80 \mathrm{~mL} \cdot \mathrm{min}^{-1} \cdot \mathrm{g}^{-1}$ catalyst, respectively. The modified HNTs with the activation energy of $30.41 \mathrm{~kJ} \cdot \mathrm{mol}^{-1}$, enthalpy of $27.93 \mathrm{~kJ} \cdot \mathrm{mol}^{-1}$, entropy of $-163.27 \mathrm{~J} \cdot \mathrm{mol}^{-1} \cdot \mathrm{K}^{-1}$ and catalytic activity of $91 \%$.In addition, the modified HNTs catalysts have higher efficiency than the common $\mathrm{H}_{2}$ generation rate which only keep $220.5 \mathrm{~mL} \cdot \mathrm{min}^{-1} \mathrm{~g}^{-1}$ catalyst.

The catalyze system (HNTs-APTMS-Mo-SL) has been synthesized by APTMS grafted on the HNTs and self-assembly way [53]. The results revealed that the functionalized catalyst could be filtered and maintain high-activity to catalyze the alkene epoxidation. It is hardly loss catalytic activity even though repeated for at least eight times.The catalyst easily to converted the active material such as the linear, aromatic alkenes and cyclic, and recycled in the catalyze reaction system. It is indicated that the catalytic mechanism of functionalized catalyst composited the Mo salen for epoxidation could be concluded for the interact bonding between Mo and the salen ligands.

\section{Conclusion and future applications.}

In this review, we focused on summarize the recent advance about modified HNTs. Key concerns, in field of nanoposites, catalysts, adsorbent and drug delivery system. Although the modified HNTs have obtained a lot of extraordinary achievement in various fields, such as biomedical application, industrial catalyst, nano composite filler and tissue engineering scaffolds, the surface utilization percentage 
and the transport pathway and uptake mechanism in the vivo also the significant challenges needed to further research.

\section{Conflict ofinterests}

The authors declare that they have no conflicts of interest to this work.

\section{Reference}

1. Pasbakhsh P, Churchman G J, Keeling J L. Characterisation of properties of various halloysites relevant to their use as nanotubes and microfibre fillers. [J]. Applied Clay Science, 2013, 74: 47-57.

2. Viviana Vergaro, Yuri M. Lvov, Stefano Leporatti. Halloysite clay nanotubes for resveratrol delivery to cancer cells. [J]. Macromolecular Bioscience, 2012, 12 (9): $1265-1271$

3. Xiumei Sun, Yao Zhang, Hebai Shen, Nengqin Jia. Direct electrochemistry and electrocatalysis of horseradish peroxidase based on halloysite nanotubes/chitosan nanocomposite film. [J]. Electrochimica Acta , 2010 , 56 (2) : 700-705

4. Hashemifard S A, Ismail A F, Matsuura T. Mixed matrix membrane incorporated with large pore size halloysite nanotubes (HNT) as filler for gas separation: experimental. [J]. Journal of colloid and interface science, 2011, 359 (2): 359-370.

5. Parthajit Pal, Mrinal Kanti Kundu, Asish Malas, Chapal Kumar Das. Compatibilizing Effect of Halloysite Nanotubes in Polar-NonpolarHybrid System. [J]. applied polymer science, 2013, 395, 87, 1-7

6. Zhi Li, Daniel Fernández Expósito, Alejandro Jiménez González, De-Yi Wang. Natural halloysite nanotube based functionalized nanohybridassembled via phosphorus-containing slow release method: Ahighly efficient way to impart flame retardancy to polylactide. [J]. European Polymer Journal. 2017. 93, 458-470

7. Nalinkanth G.Veerabadran, Ronald R. Price,Yuri M. Lvov. Clay nanotubes for encapsulation and sustained release of drugs. [J]. Nano, 2007, 2(02): 115-120

8. Viviana Vergaro, Elshad Abdullayev, Yuri M. Lvov, Andre Zeitoun, Roberto Cingolani, Ross Rinaldi and Stefano Leporatti. Halloysite Clay Nanotubes: Characterization, Biocompatibility and Use as Drug Carriers. [J]. Nanotech, 2014, 
11(9): 395-396

9. Dmitry G. Shchukin, S. V. Lamaka,K. A. Yasakau M. L. Zheludkevich, Ferreira M. G.S. and Molhwald H. Active Anticorrosion Coatings with Halloysite Nanocontainers. [J]. Journal of Physical Chemistry C, 2008, 112 (4): 958-964

10. Gölnur I. Fakhrullina, Farida S. Akhatova, Yuri M. Lvov and Rawil F. Fakhrullin. Toxicity of halloysite clay nanotubes in vivo: a Caenorhabditis elegans study. [J]. Environmental Science Nano, 2014, 2 (1) : 54-59

11. M. Massaro, R. Amorati, G. Cavallaro, S. Guernelli, G. Lazzara, S. Milioto, R. Noto, P. Pomad, S. Riela. Direct chemical grafted curcumin on halloysite nanotubes asdual-responsive prodrug for pharmacological applications. [J]. Colloids and Surfaces B Biointerfaces. 2016, $140:$ 505-513

12. Frost RL and Shurvell HF, Raman Microprobe Spectroscopy of Halloysite. [J]. Clays and Clay Miner, 1997, 45, 68-72.

13. Marina Kryuchkova, Anna Danilushkina, Yuri Lvov and Rawil Fakhrullin. Evaluation of toxicity of nanoclays and graphene oxide in vivo: a Paramecium caudatum study. [J]. Environmental Science Nano, 2016, 3 (2) : 442-452

14. Yuri Lvov, Elshad Abdullayev. Functional polymer-clay nanotube composites with sustained release of chemical agents. [J]. Progress in Polymer Science, 2013, 38 (1011) : 1690-1719

15. Vahdat Vahedi, Pooria Pasbakhsh. Instrumented impact properties and fracture behaviour of epoxy/modified halloysite nanocomposites. [J]. Polymer Testing. 2014, 39: 101-114.

16. Guangyong Zeng, Zhongbin Ye, Yi He, Xi Yang, Jing Ma, Heng Shi, Ziliang Feng. Application of dopamine-modified halloysite nanotubes/PVDF blend membranes for direct dyes removal from wastewater. [J]. Chemical Engineering Journal, 2017, 323: $572-583$

17. A.M. Carrillo, J.G. Carriazo. $\mathrm{Cu}$ and $\mathrm{Co}$ oxides supported on halloysite for the totaloxidation of toluene. [J]. Applied Catalysis B Environmental. 2015, 164 (4): 443 452

18. Yong Lin, Kai Mo Ngb, Chi-Ming Chan, Guoxing Sun, Jingshen Wu. High-impact 
polystyrene/halloysite nanocomposites prepared by emulsion polymerization using sodium dodecyl sulfate as surfactant. [J]. Journal of Colloid and Interface Science, 2011, $358(2): 423-9$

19. Wang Jinhua, Zhang Xiang, Zhang Bing, Zhao Yafei, Zhai Rui, Liu Jindun, Chen Rongfeng. Rapid adsorption of Cr (VI) on modified halloysite nanotubes. [J]. Journal of Colloid and Interface Science, 2011, 358 (2): 423-9

20. Baochun Guo,Quanliang Zou, Yanda Lei and Demin Jia. Structure and Performance of Polyamide 6/Halloysite Nanotubes Nanocomposites. [J]. Polymer Journal, 2009, 41 (10): 835-842

21. Xinyi Wan, Yingqing Zhan, Guangyong Zeng, Yi He. Nitrile functionalized halloysite nanotubes/poly (arylene ether nitrile) nanocomposites : Interface control, characterization and improved properties. [J]. Applied Surface Science, 2017, 393:110

22. Youhong Tang, Shiqiang Deng, Lin Ye, Cheng Yang, Qiang Yuan, Jianing Zhang, Chengbi Zhao. Effects of unfolded and intercalated halloysites on mechanical properties of halloysite-epoxy nanocomposites. [J]. Composites: Part A 2011, 42 (4) :345-354

23. Shiqiang Deng, Jianing Zhang, Lin Ye. Halloysite-epoxy nanocomposites with improved particle dispersion through ball mill homogenisation and chemical treatments. [J]. Composites Science and Technology, 2009, 69 (14): 2497-2505

24. Xiaoyu Li, Qian Yang, Jing Ouyang, Huaming Yang, Shi Chang. Chitosan modified halloysite nanotubes as emerging porous microspheres for drug carrier. [J]. Applied Clay Science, 2016, 126: 306-312

25. Mingxian Liu, Libing Dai, Huizhe Shi, Sheng Xiong, Changren Zhou.In vitro evaluation of alginate/halloysite nanotube composite scaffolds for tissue engineering. [J]. Materials Science and Engineering C, 2015, 49: 700-172

26. C. Liu, Y. F. Luo, Z. X. Jia et al. Enhancement of mechanical properties ofpoly(vinyl chloride) with polymethyl methacrylate-graftedhalloysite nanotube. [J]. Express Polymer Letters, 2011, 5 (7): 591-603

27. Cuiping Li,Jiguang Liu,Xiaozhong Qu. Polymer-Modified Halloysite Composite 
Nanotubes. [J]. Journal of Applied Polymer Science, 2010, 110 (6): 3638-3646

28.E.Bischoff,D.A.Simon,H.S.Schrekker,M.Lavorgna,L.Ambrosio,S.A.Liberman,R.S. Mauler. Ionic liquid tailored interfaces in halloysite nanotube/heterophasic ethylenepropylene copolymer nanocompositeswith enhanced mechanical properties. [J]. European Polymer Journal, 2016, 82: 82-92

29. Yanda Lei, Zhenghai Tang, Lixin Zhu, Baochun Guo, Demin Jia. Functional thiol ionic liquids as novel interfacial modifiers in SBR/HNTs composites. [J]. Polymer. 2011, 52 (5) : 1337-1344

30. H. Ismail, P. Pasbakhsh, M. N. A. Fauzi, A. Abu Bakar. Morphological, thermal and tensile properties of halloysite nanotubes filled ethylene propylene diene monomer(EPDM) nanocomposites. [J]. Polymer. Testing. 2008, 27 (7): 841-850.

31. Reyhaneh Berahman, MaryamRaiati, Majid Mehrabi Mazidi, Seyed Mohamad Reza Paran. Preparation and characterization of vulcanized silicone rubber/ halloysite nanotube nanocomposites: Effect of matrix hardness and HNT content. [J]. Materials and Design, 2016, 104: 333-345

32. Wenyi Kuang, Zhijun Yang,Zhenghai Tang, Baochun Guo. Wrapping of polyrhodanine onto tubular clay and its prominent effects on the reinforcement of the clay for rubber. [J]. Composites Part A Applied Science and Manufacturing. 2016, 84: $344-353$

33. Muhammad JawwadSaif, Muhammad Naveed, Khalid Mahmood Zia, Muhammad Asif. Pristine and $\gamma$-irradiated halloysite reinforced epoxy nanocomposites-Insight study. [J]. Radiation Physics and Chemistry, 2016, 127: 115-121

34. S.Barrientos-Ramírez, E. V. Ramos-Fernández, J. Silvestre-Albero, A. SepúlvedaEscribano, M. M. Pastor-Blas, A. González-Montiel. Use of nanotubes of natural halloysite as catalyst support in the atom transfer radical polymerization of methyl methacrylate. [J]. Microporous and Mesoporous Materials, 2009, 120 (1-2): 132-140 35. Viviana Vergaro, Elshad Abdullayev, Yuri M. Lvov, Andre Zeitoun, Roberto Cingolani, Ross Rinaldi, and Stefano Leporatti. Cytocompatibility and Uptake of Halloysite Clay Nanotubes. [J]. Biomacromolecules. 2010, 11 (3): 820-826

36. Raghuvara Yendluri,Daniel P. Otto,Melgardt M. De Villiersb, Vladimir Vinokurov, 
Yuri M.Lvov. Application of halloysite clay nanotubes as a pharmaceutical excipient. [J]. Int J Pharm, 2017, 521 (1-2): 267-273

37. Elshad Abdullayev, Anupam Joshi, Wenbo Wei, Yafei Zhao, and Yuri Lvov. Enlargement of halloysite clay nanotube lumen by selective etching of aluminum oxide. [J]. Acs Nano. 2012, 6 (8): 7216-7226

38. Weng On Yah,Atsushi Takaharaand Yuri M. Lvov. Selective Modification of Halloysite Lumen withOctadecylphosphonic Acid: New Inorganic Tubular Micelle. [J]. Journal of the American Chemical Society. 2012, 134 (3):1853-9

39. Hui Li, Xiaohong Zhu, Jiangfeng Xu, Wei Peng, Shian Zhong and Yan Wang. The combination of adsorption by functionalized halloysite nanotubes and encapsulation by polyelectrolyte coatings for sustained drug delivery. [J]. RSC Advances. 2016, 6(59): 54463-54470.

40. Daoyong Tan, Peng Yuan, Faïza Annabi-Bergaya, Dong Liu, LinjiangWang, Hongmei Liu, Hongping He. Loading and in vitro release of ibuprofen in tubular halloysite. [J]. Applied clay science, 2014, 96: 50-55.

41. Daoyong Tan, Peng Yuan, Faïza Annabi-Bergaya, Huaguang Yu, Dong Liu, Hongmei Liu, Hongping He. Natural halloysite nanotubes as mesoporous carriers for the loading of ibuprofen. [J]. Microporous and Mesoporous Materials, 2013, 179: 8998

42. Sandip Rooj, Amit Das, Varun Thakur, R.N. Mahaling, Anil K. Bhowmick, Gert Heinrich. Preparation and properties of natural nanocomposites based on natural rubberand naturally occurring halloysite nanotubes. [J]. Materials and Design. 2010, $31,2151-2156$

43. Peng Luo, Yafei Zhao, Bing Zhang, Jindun Liu, Yong Yang,Junfang Liu. Study on the adsorption of Neutral Red from aqueous solution onto halloysite nanotubes. [J]. Water Research. 2010, 44 (5): 1489-1497

44. Ruijun Li, Qun He, Zheng Hu, Shengrui Zhang, Lijun Zhang, Xijun Chang. Highly selective solid-phase extraction of trace $\operatorname{Pd}(\mathrm{II})$ by murexide functionalized halloysite nanotubes. [J]. Analytica Chimica Acta, 2012, 713 (3) :136-144 45. Wang Jinhua, Zhang Xiang, Zhang Bing, Zhao Yafei, Zhai Rui, Liu Jindun, Chen 
Rongfeng. Rapid adsorption of $\mathrm{Cr}$ (VI) on modified halloysite nanotubes. [J]. Desalination. 2010, 259 (1-3): 22-28

46. Zhitao Wang, Huixian Wang, Jindun Liu, Yatao Zhang. Preparation and antifouling property of polyethersulfone ultrafiltration hybrid membrane containing halloysite nanotubes grafted with MPC via RATRP method. [J]. Desalination, 2014, 344 (344): 313-320

47. Yuanyuan Zhang, Rui Gao, Min Liu, Baoming Shi,Anshan Shan, Baojing Cheng. Use of modified halloysite nanotubes in the feed reduces the toxic effects of zearalenone on sow reproduction and piglet development. [J]. Theriogenology, 2015, 83 (5) : 932

48.Yuanyuan Zhang, Rui Gao, Min Liu, Changjiang Yan and Anshan Shan. Adsorption of modified halloysite nanotubes in vitro and the protective effect in rats exposed to zearalenone. [J] .Archives of Animal Nutrition, 2014, 68 (4): 320-335

49. S. Barrientos-Ramirez, G. Montes de Oca-Ramirez, E. V. Ramos-Fernandez, A. Sepulveda-Escribano, M. M. Pastor-Blas, A. Gonzalez-Montielb. Surface modification of natural halloysite clay nanotubes with aminosilanes. Application as catalyst supports in the atom transfer radical polymerization of methyl methacrylate. [J]. Applied Catalysis A General, 2011, 406 (1): 22-33

50. Zhongkui Zhao, Jinfeng Ran, Yanhua Jiao, Weizuo Li, Boyuan Miao. Modified natural halloysite nanotube solely employed as an efficient and low-cost solid acid catalyst for alpha-arylstyrenes production via direct alkenylation. [J]. Applied Catalysis A General, 2016, 513: 1-8

51. Jing Ouyang, Zai Zhao, Yi Zhang, Huaming Yang. Textual properties and catalytic performances of halloysite hybrid $\mathrm{CeO}_{2}-\mathrm{ZrO}_{2}$ nanoparticles. [J].Journal of Colloid and Interface Science, $2017,505: 430$

52. Nurettin Sahiner, Sultan Butun Sengel.Environmentally benign halloysite clay nanotubes as alternative catalyst to metal nanoparticles in $\mathrm{H}^{2}$ production from methanolysis of sodium borohydride. [J]. Fuel Processing Technology, 2017, 158: 1-8 53.Yu Long, Bing Yuan, Jiantai Ma. Epoxidation of alkenes efficiently catalyzed by Mo salen supported on surface-modified halloysite nanotubes. [J]. Chinese Journal of 
Catalysis, 2015, 36 (3): 348-354 